\title{
ESTRATÉGIAS PARA AVALIAÇÃO DO DESEMPENHO CLÍNICO DE ESTUDANTES DE ODONTOLOGIA
}

\author{
STRATEGIES FOR EVALUATING DENTISTRY \\ STUDENTS' CLINICAL PERFORMANCE \\ Patricia Valente Araújo Jacques Gonçalves ${ }^{1}$,
}

Henrique Pretti ${ }^{2}$, Karina Imaculada Rosa Teixeira ${ }^{3}$,

Cláudia Silami Magalhães ${ }^{4}$, Allyson Nogueira Moreira ${ }^{5}$,

${ }^{1}$ Universidade

Federal de Minas

Gerais (UFMG), Belo

Horizonte, Brasil.

${ }^{2}$ Universidade

Federal de Minas

Gerais (UFMG), Belo

Horizonte, Brasil.

${ }^{3}$ Fundação Fead

Minas, Belo Horizonte,

Brasil.

${ }^{4}$ Universidade

Federal de Minas

Gerais (UFMG), Belo

Horizonte, Brasil.

${ }^{5}$ Universidade

Federal de Minas

Gerais (UFMG), Belo

Horizonte, Brasil.

${ }^{6}$ Universidade

Federal de Minas

Gerais (UFMG), Belo

Horizonte, Brasil.

Rogéli Tibúrcio Ribeiro da Cunha Peixoto ${ }^{6}$

\section{RESUMO}

A avaliação do desempenho clínico de estudantes de Odontologia é muito importante para motivar o aprendizado. Este artigo propõe-se a apresentar uma revisão de literatura sobre métodos de avaliação de desempenho clínico e a desenvolver um protocolo para ser aplicado nas disciplinas do curso de Odontologia da UFMG. O Protocolo de Avaliação Clínica (PAC) foi elaborado a partir do Objective Structured Clinical Examination (OSCE) e do Structured Clinical Operative Test (SCOT), métodos de avaliação mais encontrados na literatura, com a finalidade de realizar uma avaliação objetiva do aprendizado do aluno em pontos relacionados a anamnese, diagnóstico, plano de tratamento, controle de infecção, técnicas, habilidade, comunicação e profissionalismo. A autoavaliação realizada pelos alunos também faz parte desse protocolo, sendo interessante para se diagnosticarem pontos fracos e virtudes dos estudantes. O PAC pode ser uma 
alternativa aos métodos convencionais de avaliação, reduzindo a subjetividade e tornando o processo avaliativo mais confiável e homogêneo.

Palavras-chave: Avaliação educacional. Educação em Odontologia. Autoavaliação. Estudantes. Competência clínica.

\section{INTRODUÇÃO}

A competência clínica e a qualidade dos profissionais de saúde são objeto de intensas pesquisas no campo da avaliação (PRESCOTT et al., 2002; BOWERS, 2006). Especificamente para o curso de Odontologia, a avaliação do desempenho clínico dos estudantes torna-se essencial, pois eles realizam procedimentos clínicos invasivos nos pacientes ao longo do curso de graduação.

Segundo Mossey et al. (2001), a avaliação clínica é crucial no processo de aprendizado de estudantes. Esses autores apontam falhas nos métodos de avaliação clínica tradicional: (a) uma grande quantidade de pacientes apresenta graus diferentes de necessidades e é atendida por alunos diferentes, e (b) a subjetividade do examinador, que resulta em uma variação interexaminador na avaliação de um mesmo procedimento. Manogue et al. (2001) também perceberam que a falta de consistência e objetividade na avaliação é um problema comum detectado pelos departamentos quando são consideradas as avaliações práticas em dentística restauradora. 
Técnicas operatórias e habilidades manuais não são os únicos aspectos a serem avaliados nos procedimentos clínicos. Vários campos da competência, como, por exemplo, comunicação, conhecimento e organização, devem ser considerados, além da habilidade técnica. Diferentes métodos de avaliação clínica têm sido propostos na literatura, na tentativa de melhorar esse processo, englobando todos os pontos relevantes para a prática clínica e, também, a fim de torná-lo mais objetivo e consistente.

A avaliação é denominada formativa se há evidência de que ela é utilizada para melhorar a aprendizagem. Na avaliação formativa, os professores devem se informar sobre o progresso dos alunos para se adaptarem às necessidades deles. Esse tipo de avaliação tem como base a cultura do sucesso, ou seja, todos os alunos são capazes de aprender. Para que a avaliação formativa tenha êxito, não basta que apenas alguns passos sejam acrescentados, são necessários investimentos nas várias atividades de ensino e nas crenças do professor. É necessária uma cultura de questionamentos e reflexão, na qual os alunos aprendem a partir das discussões compartilhadas com professores e colegas (BLACK; WILLIAM, 1998).

Para isso, além de avaliar a habilidade clínica e a competência dos estudantes, a autoavaliação dos alunos também pode ser incorporada. A autoavaliação e a posterior discussão com o professor são instrumentos válidos para melhorar a percepção do aluno com relação ao próprio desempenho. De acordo com Zartman et al. (2002), essa abordagem aumenta a autoconfiança do aluno e favorece o aprendizado. 
Para que o aluno se autoavalie, é necessário que esse tenha conhecimento da meta desejada, da própria posição presente e ideia do caminho para sair da posição presente e alcançar a meta desejada (DECY; RYAN, 1994; FREITAS, 1999).

Manogue et al. (2001) afirmam que os estudantes de Odontologia necessitam desenvolver habilidades, atitudes e conhecimento suficientes para realizarem atendimento clínico invasivo ainda durante a graduação. A avaliação do desempenho clínico desses estudantes torna-se essencial tanto para assegurar um atendimento adequado para o paciente quanto para motivar o aluno a dar continuidade ao aprendizado.

Diante disso, o objetivo deste estudo é apresentar uma breve revisão sobre os principais métodos de avaliação do desempenho clínico encontrados na literatura. Além disso, um método de avaliação clínica dos alunos, o Protocolo de Avaliação Clínica (PAC), foi elaborado a partir dos dados encontrados na literatura e será aqui apresentado como alternativa aos métodos convencionais de avaliação atualmente empregados.

\section{MÉTODOS DE AVALIAÇÃO DO DESEMPENHO CLÍNICO}

Harden e Glesson (1979) desenvolveram a Avaliação Clínica Objetiva Estruturada (Objective Structured Clinical Examination - OSCE), como um método para avaliação objetiva da competência, a fim de reduzir a subjetividade do examinador e do paciente. Nesse método, situações clínicas são simuladas em manequins e todos os alunos são avaliados em relação 
aos mesmos procedimentos. Bancadas de trabalho, com manequins para simular situações clínicas, são montadas, e, com isso, as únicas variáveis avaliadas são o conhecimento e a habilidade do candidato. Os OSCE têm sido utilizados em avaliações de diagnóstico e planos de tratamento, mas falham em avaliar outras habilidades clínicas.

Segundo Graham, Bitzer e Anderson (2013), o OSCE é uma abordagem para a avaliação da competência que avalia a resposta de um aluno quando ele é confrontado com um cenário clínico. Habilidades críticas para profissionais de saúde, como a perspicácia de diagnóstico e a tomada de decisão clínica, são avaliadas. Esse método de avaliação não se destina a medir a aquisição de conhecimento pontual, ela objetiva medir a capacidade do estudante em sintetizar informações e aplicar o conhecimento clínico em várias estações que simulam uma situação clínica.

Segundo Mossey et al. (2001), embora o OSCE aumente a objetividade, a falta de autenticidade na avaliação de habilidades interpessoais e no manejo do comportamento são os maiores problemas encontrados. Após a avaliação do método, os autores concluíram que ele apresentou falhas fundamentais, pois a habilidade clínica operatória em odontologia não deveria ser avaliada utilizando-se cenários e modelos irreais. O OSCE foi desenvolvido para ser aplicado na medicina, e há uma diferença fundamental entre avaliar um estudante de Odontologia e um de Medicina, pois é suficiente que um graduando em Medicina tenha conhecimentos de diagnóstico e interpretação de situações clínicas. Por outro lado, o estudante de Odontologia necessita, além disso, ser 
um competente cirurgião, pois, ainda durante a graduação, já realiza procedimentos invasivos nos pacientes.

Zartman et al. (2002) acompanharam a implementação do OSCE como um método para a avaliação de estudantes no Departamento de Odontopediatria do Baylor College of Dentistry e o impacto dele sobre o ensino. Os autores concluíram que o OSCE é um teste que consome muito tempo e necessita de infraestrutura satisfatória, como um espaço físico suficiente para que as bancadas de trabalho possam ser montadas. Porém, mostrou-se um método satisfatório para a avaliação do desempenho e da evolução dos estudantes ao longo do curso.

Major (2005) afirma que o OSCE tem como proposta original a avaliação de habilidades clínicas adquiridas por estudantes de medicina. É um método de avaliação objetivo, em que as variáveis relacionadas ao paciente são eliminadas e todos os alunos são avaliados em relação aos mesmos procedimentos.

De acordo com Mossey e Newton (2001), métodos como o Structured Clinical Operative Test (SCOT) têm sido indicados para complementar o OSCE na avaliação de procedimentos clínicos invasivos. Conforme os parâmetros do SCOT, o estudante realiza procedimentos clínicos autênticos em pacientes reais, sob supervisão, e será avaliado a partir de critérios preestabelecidos em um checklist. Imediatamente após a avaliação, o desempenho é discutido entre examinador e estudante de maneira construtiva. Claramente, esse sistema não é inteiramente objetivo devido às variações entre os pacientes, mas representa um cenário clínico autêntico, o que o torna um método de avaliação muito válido. A objetividade 
pode ser maximizada com mudanças cuidadosas nas questões examinadas e com o planejamento meticuloso do checklist utilizado. Enquanto o OSCE é extremamente satisfatório ao avaliar a competência do aluno em relação ao diagnóstico e ao plano de tratamento, o SCOT pode ser utilizado para avaliar as habilidades manuais durante um procedimento clínico em pacientes reais.

Yip et al. (2001), que descreveram uma abordagem de ensino baseado na competência, atestam que esse método utiliza critérios que objetivam refletir a organização, o relacionamento com o paciente e o trabalho clínico em todos os aspectos do tratamento do indivíduo durante as sessões clínicas. A autorreflexão clínica dá oportunidade aos estudantes de repensarem e avaliarem o próprio desempenho, imediatamente após o teste. A educação baseada na competência tem as seguintes vantagens: reduzir a dependência de aulas expositivas, melhorar o desempenho dos estudantes por meio da participação ativa na aprendizagem, encorajar a autoavaliação crítica da competência teórica, integrando esses conhecimentos à prática, e incentivar a interdisciplinaridade no ensino. Isso permite que os professores monitorem mais efetivamente o progresso clínico dos alunos ao longo do curso de graduação em Odontologia. Dessa forma, o ensino seria visto como integral e holístico, ao contrário do modelo tradicional de ensino fragmentado em disciplinas, que predispõe os alunos a um ensino mecanicista.

Manogue et al. (2001) avaliaram, por meio de questionários enviados a professores, quais eram os principais métodos de avaliação do desempenho dos alunos nas clínicas de graduação 
de universidades do Reino Unido. O método de avaliação mais comumente utilizado foi a observação diária dos alunos seguida de um julgamento implícito por parte dos professores, sem critérios predefinidos ou exigência da realização de um número mínimo de procedimentos clínicos, utilizando-se a produção na clínica como medida da competência dos alunos. Os autores concluíram que o momento é oportuno para se repensar as estratégias de avaliação da competência clínica de estudantes de Odontologia.

Para Macluskey et al. (2004), tornar-se competente requer mais que o conhecimento básico necessário para iniciar a prática em disciplinas odontológicas, mas envolve conhecimento, atitude e habilidade prática do estudante. É desejável que o acesso à formação aumente a autoconfiança e encoraje a autoavaliação, melhorando a aprendizagem e o desempenho clínico. Esses autores avaliaram se o SCOT pode ser utilizado como uma ferramenta de avaliação do desempenho clínico durante uma exodontia. Os examinadores consideraram o SCOT uma alternativa viável para a avaliação clínica, pois esse reduz a subjetividade dos exames. Porém, relataram que é um método muito trabalhoso e demorado, prejudicando o andamento da clínica e dificultando a supervisão de outros estudantes. O SCOT também obteve boa aceitação entre os estudantes.

De acordo com Luckesi (1998), na prática de aferição do aproveitamento escolar, os professores realizam, basicamente, três procedimentos sucessivos: medida do aproveitamento escolar, transformação da medida em nota ou conceito e utilização dos resultados identificados. Os resultados 
da aprendizagem são obtidos pela medida, variandose a especificidade e a qualidade dos mecanismos e dos instrumentos utilizados para obtê-la. Com isso, a aferição da aprendizagem escolar é utilizada, na maioria das vezes, para classificar os alunos em aprovados ou reprovados.

Prescott et al. (2002) e Suebnukarn e Haddawy (2006) descreveram alguns dos principais pontos-chave relatados por educadores e clínicos envolvidos no desenvolvimento de sistemas de avaliação baseados na competência para estudantes da área de saúde. São eles: a avaliação de habilidades em diferentes áreas de competência, a associação da avaliação com o treinamento e o acompanhamento do desempenho ao longo do curso. Os autores propõem um método de avaliação denominado Longitudinal Evaluation of Performance (LEP), o qual utiliza a observação direta do aluno na prática clínica e conta com o julgamento do examinador em relação ao desempenho em várias categorias. A avaliação regular ao longo do semestre permite que problemas no desempenho sejam identificados. Um planejamento apropriado para remediá-los pode ser colocado em prática, dirigindo o aprendizado do aluno em direção a um padrão de competência requerido.

Gonçalves e Verdi (2005) propuseram-se a identificar as questões éticas relacionadas ao atendimento de pacientes em uma clínica odontológica de ensino, por meio de questionários feitos com professores do curso de Odontologia de uma universidade pública federal. Os autores perceberam que as pessoas em busca de tratamento nessas clínicas tornam-se frequentemente vulneráveis. Concluem que a formação da 
competência ética de futuros profissionais e a postura desses frente aos pacientes atendidos nas clínicas de ensino são responsabilidade de todo o corpo docente.

\section{CONSIDERAÇÕES SOBRE O PROCESSO DE APRENDIZAGEM - ELABORAÇÃO DE UM NOVO MÉTODO DE AVALIAÇÃO}

A prática educacional brasileira opera, na quase totalidade das vezes, como verificação. Portanto, tem sido incapaz de retirar do processo de aferição as consequências mais significativas para a melhoria da qualidade e do nível de aprendizagem dos educandos, uma vez que a nota atribuída ao aluno torna-se mais importante que o aprendizado. A verificação transforma o processo dinâmico da aprendizagem em passos estáticos e definitivos. A avaliação, ao contrário, manifesta-se como um ato dinâmico, que qualifica e subsidia o reencaminhamento da ação, possibilitando consequências na direção da construção dos resultados que se deseja. O primeiro objetivo da aferição do aproveitamento escolar não deve ser a aprovação ou a reprovação do educando, mas o direcionamento da aprendizagem e o consequente desenvolvimento do estudante. A avaliação subsidia decisões a respeito da aprendizagem, destinando-se a construir com os e nos educandos conhecimentos, habilidades e hábitos que possibilitem a eles o efetivo desenvolvimento, por meio da assimilação ativa do legado cultural da sociedade. Para que se utilize corretamente a avaliação no processo ensino-aprendizagem, importa estabelecer um padrão mínimo de conhecimentos, habilidades 
e hábitos, e não uma média mínima de notas, como ocorre hoje na prática escolar (LUCKESI, 2001).

Gaião et al. (2001) descrevem duas formas de se conceber a educação: a abordagem tradicional e a abordagem sóciopolítico-cultural. $\mathrm{Na}$ abordagem tradicional, a relação professor-aluno é do tipo vertical e o processo de avaliação tem como objetivo a exatidão da reprodução do conteúdo comunicado em sala de aula. Numa abordagem sócio-políticocultural, o papel do professor desponta como sendo não o de ensinar, mas o de ser responsável pela facilitação do processo de aprendizagem por parte do aluno. O professor não está mais num nível superior ao aluno, demonstrando poder; ele assume uma postura mais objetiva e direta, deixando o próprio aluno como o sujeito do próprio conhecimento. O trabalho adquire, assim, caráter coletivo. A avaliação do aluno deve merecer, por parte do professor, toda a atenção possível, pois é por meio dela que se chega à conclusão sobre a utilidade ou não dos esforços despendidos. Nessa abordagem, a avaliação do processo ensino-aprendizagem considerada verdadeira consiste na autoavaliação e/ou na avaliação mútua e permanente da prática educativa por professor e alunos. Tanto os alunos quanto os professores devem saber quais são as próprias dificuldades e progressos. O objetivo principal é criar condições para que se desenvolva no aluno uma atitude de reflexão crítica.

Considerando-se pontos importantes do OSCE e do SCOT, um Protocolo de Avaliação Clínica (PAC) foi elaborado, a fim de ser empregado como instrumento de avaliação diária dos alunos que realizam atendimento clínico a pacientes sob a supervisão 
de docentes. Essa avaliação envolveu as principais áreas de competência das disciplinas de graduação em Odontologia do Departamento de Odontologia Restauradora da Faculdade de Odontologia da Universidade Federal de Minas Gerais (FOUFMG). O protocolo proposto teve como finalidade realizar uma avaliação objetiva do aprendizado do aluno em pontos relevantes relacionados a anamnese, diagnóstico, plano de tratamento, controle de infecção, técnicas, habilidade manual, comunicação e profissionalismo. O PAC consistiu-se de um checklist composto por quarenta critérios de avaliação. Para cada critério, foi atribuído um valor de acordo com uma escala de três pontos: insatisfatório (1), regular (2) e satisfatório (3). Quando um determinado critério não fazia parte da avaliação, a opção "não se aplica" era assinalada, e o critério era, então, excluído da soma final. A totalização da pontuação resultava da soma dos valores atribuídos a cada critério, dividida pelo número de critérios avaliados, variando de zero a três, em que o valor três representa $100 \%$ de rendimento.

O objetivo da implementação desse método de avaliação é reduzir a subjetividade da avaliação do desempenho clínico dos alunos, uma vez que todos os professores passam a considerar os mesmos critérios. Porém, algumas dificuldades são esperadas. Primeiramente, pode-se ressaltar o tempo gasto para a avaliação diária dos alunos. Assim como Zartman et al. (2001), que relataram que testes para avaliação do desempenho clínico consomem muito tempo do examinador, o fator tempo pode se tornar um dificultador no processo de avaliação. À medida que os professores forem se habituando a um novo formato de avaliação, esse fator deve, aos poucos, tornar-se menos relevante. 
A relação professor-aluno também pode ser considerada outro fator dificultador na implementação desse método de avaliação, pois os professores são frequentemente solicitados pelos outros alunos e, muitas vezes, não conseguem acompanhar adequadamente aquele que está sendo avaliado. Com isso, nem sempre todas as etapas do tratamento são corretamente observadas. Embora o Ministério da Educação e Cultura (MEC) preconize uma relação de cinco alunos para cada professor em clínicas de graduação no curso de Odontologia, essa relação nem sempre pode ser alcançada. Ainda que as exigências do MEC sejam respeitadas, há dificuldade de se conseguir acompanhar o aluno durante todos os passos operatórios realizados.

De acordo com Mannix et al. (2006), o relacionamento aluno-professor é um fator muito importante no processo de aprendizagem. A boa relação desenvolvida ao longo do tempo aperfeiçoa potencialmente o aprendizado clínico do aluno. Além disso, a familiaridade facilita e torna mais prazeroso o tempo despendido durante as aulas, os alunos tornam-se mais organizados, sendo capazes de refletir esses princípios na prática clínica.

Outro ponto a ser observado diz respeito à percepção da prática clínica pelo aluno, a ser realizada pelas autoavaliações. Um ponto interessante nesse processo pode ser a possibilidade de aluno e professor discutirem os pontos positivos e negativos de um determinado procedimento, fazendo com que os discentes aprendam mais com os erros que cometerem. Não basta simplesmente atribuir um conceito ou uma nota a um aluno, classificando-o como apto ou não a prosseguir no curso. 
O importante é que ele aprenda a enxergar os erros cometidos, tendo postura crítica diante dos procedimentos executados. De acordo com Gaião et al. (2001), o professor deve despontar não apenas com o papel de ensinar, mas também com o de ajudar o aluno a aprender. Não basta transmitir informações, também se devem criar condições para que o aluno as adquira. Nesse sentido, o educador desponta como o mediador do diálogo do aluno com o conhecimento, valorizando a seriedade na busca desse conhecimento, resgatando o rigor científico.

Além da preocupação com o aprendizado do aluno, é importante enfatizar os aspectos éticos relacionados ao atendimento clínico de pacientes. Muitas vezes, as rotinas adotadas nas clínicas de ensino visam muito mais a formação do profissional e o cumprimento da produção acadêmica, em detrimento da real necessidade de tratamento do paciente.

Segundo Fortes, citado por Gonçalves e Verdi (2005), "todo ser humano, quando na posição de paciente, deve ser tratado em virtude de suas necessidades de saúde e não como um meio para a satisfação de interesses de terceiros, da ciência, dos profissionais de saúde ou de interesses industriais e comerciais" (GONÇALVES; VERDI, 2005 p. 201). O corpo docente precisa estar atento à construção da competência ética dos futuros odontólogos e à postura deles frente aos pacientes, que não devem ser vistos como simples objeto de ensino.

\section{PERSPECTIVAS}

O Protocolo de Avaliação Clínica (PAC) pode se tornar uma boa ferramenta para avaliação diária do desempenho clínico de 
alunos do curso de graduação em Odontologia, por reduzir a subjetividade da avaliação.

O passo seguinte será aplicar O PAC em disciplinas do Departamento de Odontologia Restauradora da FO-UFMG, avaliando os benefícios e as dificuldades encontradas na implementação desse novo método de avaliação.

Uma reflexão sobre os critérios atuais de avaliação do desempenho clínico dos estudantes faz-se necessária, uma vez que eles têm se mostrado muito subjetivos e variáveis de acordo com critérios definidos por cada professor.

A incorporação da autoavaliação e de um feedback com o aluno parecem ser importantes para diagnosticar pontos fracos e virtudes dos estudantes, enriquecendo a trajetória dele e encorajando-o a buscar caminhos para aprimorar cada vez mais as habilidades que possui.

\title{
PROTOCOLO DE AVALIAÇÃO CLÍNICA
}

\section{CLINICAL ASSESSMENT PROTOCOL}

\author{
Aluno: \\ Professor: \\ Disciplina: \\ Paciente:
}


1. Coleta de dados (preenchimento do prontuário odontológico)

\begin{tabular}{|c|c|c|c|c|}
\hline \multicolumn{2}{|c|}{ INSATISFATÓRIO } & REGULAR & \multicolumn{2}{|c|}{ SATISFATÓRIO } \\
\hline 1 & 2 & 3 & 4 & 5 \\
\hline
\end{tabular}

Não se aplica

2. Diagnóstico (levantamento de necessidades, exames: clínico e complementares)

\begin{tabular}{|c|c|c|c|c|}
\hline \multicolumn{2}{|c|}{ INSATISFATÓRIO } & REGULAR & \multicolumn{2}{|c|}{ SATISFATÓRIO } \\
\hline 1 & 2 & 3 & 4 & 5 \\
\hline
\end{tabular}

Não se aplica

3. Plano de tratamento (elaboração de um tratamento adequado às necessidades do paciente)

\begin{tabular}{|c|c|c|c|c|}
\hline \multicolumn{2}{|c|}{ INSATISFATÓRIO } & REGULAR & \multicolumn{2}{|c|}{ SATISFATÓRIO } \\
\hline 1 & 2 & 3 & 4 & 5 \\
\hline
\end{tabular}

Não se aplica

4. Técnica e habilidade manual:

( ) Isolamento absoluto

\begin{tabular}{|c|c|c|c|c|}
\hline \multicolumn{2}{|c|}{ INSATISFATÓRIO } & REGULAR & \multicolumn{2}{c|}{ SATISFATÓRIO } \\
\hline 1 & 2 & 3 & 4 & 5 \\
\hline
\end{tabular}

Não se aplica 
( ) Raspagem e polimento coronário

( ) Preparo e restauração

( ) Endodontia

( ) Exodontia

\begin{tabular}{|c|c|c|c|c|}
\hline \multicolumn{2}{|c|}{ INSATISFATÓRIO } & REGULAR & \multicolumn{2}{c|}{ SATISFATÓRIO } \\
\hline 1 & 2 & 3 & 4 & 5 \\
\hline
\end{tabular}

\begin{tabular}{|l|l|}
\hline & Não se aplica \\
\hline
\end{tabular}

5. Conhecimento (embasamento teórico)

\begin{tabular}{|c|c|c|c|c|}
\hline \multicolumn{2}{|c|}{ INSATISFATÓRIO } & REGULAR & \multicolumn{2}{|c|}{ SATISFATÓRIO } \\
\hline 1 & 2 & 3 & 4 & 5 \\
\hline
\end{tabular}

Não se aplica

6. Organização: biossegurança, paramentação, organização da bandeja clínica, instrumentais

\begin{tabular}{|c|c|c|c|c|}
\hline \multicolumn{2}{|c|}{ INSATISFATÓRIO } & REGULAR & \multicolumn{2}{|c|}{ SATISFATÓRIO } \\
\hline 1 & 2 & 3 & 4 & 5 \\
\hline
\end{tabular}

\begin{tabular}{|l|l|}
\hline & Não se aplica \\
\hline
\end{tabular}

7. Comunicação: relação com o paciente, com a dupla e com o professor

\begin{tabular}{|c|c|c|c|c|}
\hline \multicolumn{2}{|c|}{ INSATISFATÓRIO } & REGULAR & \multicolumn{2}{c|}{ SATISFATÓRIO } \\
\hline 1 & 2 & 3 & 4 & 5 \\
\hline
\end{tabular}

Não se aplica 
8. Profissionalismo: pontualidade, postura perante o paciente, respeito ao paciente

\begin{tabular}{|c|c|c|c|c|}
\hline \multicolumn{2}{|c|}{ INSATISFATÓRIO } & REGULAR & \multicolumn{2}{|c|}{ SATISFATÓRIO } \\
\hline 1 & 2 & 3 & 4 & 5 \\
\hline
\end{tabular}

Não se aplica

\section{REFERÊNCIAS BIBLIOGRÁFICAS}

BLACK, P.; WILLIAM, D. Assessment and classroom learning. Assessment in education. v. 5, n. 1, p. 7-73, 1998.

BOWERS, H. F. Designing quality course management systems that fosters intra-professional education. Nurse Education Today, v. 26, p. 726-731, 2006.

DECl, E. L.; RYAN, R. M. Promoting self-determined education. Scandinavian Journal of Education Research, v. 38 , n. 1, p. 3-14, 1994.

FREITAS, Z. L. Fusão de práticas de ensino e avaliação. In: II COLÓQUIO INTERNACIONAL PAULO FREIRE, Recife, 1999. Disponível em: <www.paulofreire.ufpb.br/ paulofreire>. Acesso em: 7 abr. 2007.

GAIÃO, L.; ALMEIDA, R. V. D.; PADILHA, W. W. N. Analysis of the learning assessment at the curse of odontology of the University Federal of Paraíba. Revista da Faculdade de Odontologia da UFBA, v. 22; p. 20-25, 2001.

GONÇALVES, E. R.; VERDI, M. I. M. A vulnerabilidade e o paciente da clínica odontológica de ensino. Revista Brasileira de Bioética, v.1, n. 2, p. 195-205, 2005. 
GRAHAM, R.; BITZER, L. A. Z.; ANDERSON, O. R. Reliability and predictive validity of a comprehensive preclinical OSCE in dental education. J Dent Educ., v. 77, n. 2, p. 161167, 2013.

HARDEN, R. M.; GLESSON, F. A. Assessment of clinical competence using an objective structured clinical examination (OSCE). Med Educ., v. 13, p. 41-54, 1979.

LUCKESI, C. C. Verificação ou Avaliação: o que pratica a escola? Série Ideias. São Paulo: FDE, v. 8, p. 71-80, 1998.

MACLUSKEY, M.; HANSON, C.; KERSHAW, A.; WIGHT, A. J.; OGDEN, G. R. Development of a structured clinical operative test (SCOT) in the assessment of practical ability in the oral surgery undergraduate curriculum. British Dental Journal, v. 196, n. 4, p. 225-228, 2004.

MAJOR, D. A. OSCEs - seven years on the bandwagon: the progress of an objective structured clinical evaluation program. Nurse Education Today, v. 25. p. 442-54, 2005.

MANNIX, J.; FAGA, P.; BEALE, B.; JACKSON, D. Towards suitable models for clinical education in nursing: An ongoing conversation. Nurse Education in Practice, v. 6, p. 3-11, 2006.

MANOGUE, M.; BROWN, G.; FOSTER, H. Clinical assessment of dental students: values and practices of teachers in restorative dentistry. Med Educ., v. 35, p. 364370, 2001.

MOSSEY, P. A.; NEWTON, J. P. The Structured Clinical Operative Test (SCOT) in dental competency assessment. v. 190, n. 7, p. 387-390, 2001. 
MOSSEY, P. A.; NEWTON, J. P.; STIRRUPS, D. R. Scope of the OSCE in the assessment of clinical skills in dentistry. British Dental Journal, v. 190; n. 6, p. 323-326, 2001.

PRESCOTT, L. E.; NORCINI, Z. Z.; MCKINLAY, P., RENNIE, E. Z. S. Facing the challenges of competency-based assessment of postgraduate dental training; Longitudinal Evaluation of Performance (LEP). Medical Education, v. 36, p. 92-97, 2002.

SUEBNUKARN, S.; HADDAWY, P. A bayesian approach to generating tutorial hints in a collaborative medical problem-based learning system. Artificial Intelligence in Medicine, v. 38, p. 5-24, 2006.

YIP, H. K.; SMALES, R. J.; NEWSOME, P. R. H.; CHU, F. C. S.; CHOW, T. W. Competency-based education in a clinical course in conservative dentistry. British Dental Journal, v. 191, n. 9, p. 517-522. 2001.

ZARTMAN, R. R.; MCWHORTER, A. G.; SEALE, N. S.; BOONE, W. J. Using OSCE-Based evaluation: curricular impact over time. Journal of Dental Education, v. 66, n. 12, p. 1323-1330, 2002.

\section{ABSTRACT}

Evaluating the performance of dentistry students is essential to motivate learning. This article presents a review of the literature on the main clinical performance evaluation methods available. Based on that review, this work develops a method of clinical evaluation to be applied in the Faculty of Dentistry - UFMG. The Clinical Assessment Protocol (CAP) was developed considering OSCE (Objective Structured Clinical Examination) and SCOT (Structured Clinical Operative Test) methodologies. CAP's 
purpose is to objectively assess students' learning on topics related to medical history investigation, diagnosis, treatment planning, infection control, techniques, skills, communication and professionalism. Students' self-assessment is also part of this protocol and may be interesting to identify their own weaknesses and strengths, motivating their learning process. CAP can be an alternative to conventional evaluation methods, reducing subjectivity and providing more reliability and equity to the evaluation process.

Keywords: Educational assessment. Dentistry education. Selfassessment. Students. Clinical competence.

\section{Patricia Valente Araújo Jacques Gonçalves}

Graduada em Odontologia pela Pontifícia Universidade Católica de Minas Gerais (PUC Minas), em 1996, com especialização em Dentística pela Associação Brasileira de Odontologia (ABO$M G)$, em 2000, mestrado em Odontologia pela Faculdade de Odontologia da Universidade Federal de Minas Gerais (FOUFMG), área de concentração Dentística, em 2004, e doutorado em Odontologia pela FO-UFMG, área de concentração Clínica Odontológica, em 2009. Atualmente é professora adjunta do Departamento de Odontologia Restauradora da Faculdade de Odontologia da UFMG.

patriciavalenteufmg@gmail.com 


\section{Henrique Pretti}

Graduação em Odontologia pela PUC Minas (1985), mestrado em Ortodontia pela Faculdade de Odontologia de Bauru da Universidade de São Paulo (USP), em 1991, doutorado em Odontologia pela Universidade Federal de Minas Gerais, área de concentração Clínica Odontológica (2010) e pós-doutorado na Universidade da Carolina do Norte (2013). Atualmente é diretor da FO-UFMG e professor adjunto do Departamento de Odontopediatria e Ortodontia da FO-UFMG.

bhpretti@gmail.com

\section{Karina Imaculada Rosa Teixeira}

Possui mestrado e doutorado em Clínica Odontológica pela Universidade Federal de Minas Gerais. É professora do curso de Odontologia da Fundação Fead Minas.

karinart@gmail.com

\section{Cláudia Silami Magalhães}

Graduação em Odontologia pela Universidade Federal de Minas Gerais (1986), mestrado (1997) e doutorado (2000) em Clínica Odontológica, área de concentração Dentística, pela Universidade Estadual de Campinas. Atualmente é professora associada IV do Departamento de Odontologia Restauradora da FO-UFMG. Tem experiência na área de clínica odontológica, com ênfase em dentística. É orientadora do Programa de PósGraduação em Odontologia da FO-UFMG.

silamics@yahoo.com 


\section{Allyson Nogueira Moreira}

Graduação em Odontologia pela Universidade Federal de Minas Gerais (1986), especialização em Dentística pela Faculdade de Bauru da Universidade de São Paulo (1989) e doutorado em Odontologia, área de concentração Periodontia, pela Universidad de Buenos Aires (1999). Professor titular da Universidade Federal de Minas Gerais. Atualmente, é chefe do Departamento de Odontologia Restauradora da FO-UFMG.

dangelogatil@terra.com.br

\section{Rogéli Tibúrcio Ribeiro da Cunha Peixoto}

Graduada em Odontologia pela Universidade Federal de Minas Gerais (1987), especialização em Dentística pela Faculdade de Odontologia de Bauru (FOB-USP), mestrado em Odontologia - área de concentração Dentística - pela FO-UFMG (1999) e doutorado em Odontologia - área de concentração Dentística - pela FO-UFMG (2008). Atualmente é vice-diretora da FOUFMG e professora adjunta do Departamento de Odontologia Restauradora da FO-UFMG.

rogelit@terra.com.br 
